\title{
CONF-850504--296
}

UCRL- 91956

PREPRINT

MEDENEU BY COM

OCT 25 的

\section{NUMERICAL SIMULATION OF SECONDARY ELECTRCNN ORBITS NEAR AN ELECTRON BEAM PROPAGATING IN A LOW PRESSURE GAS}

M. R. Teague and S. S. Yu

This paper was prepared for submitta? to the

1985 Particle Accelerator Conference

Vancouver, B. C., Canada

May $13-16,1985$

August 14, 1985

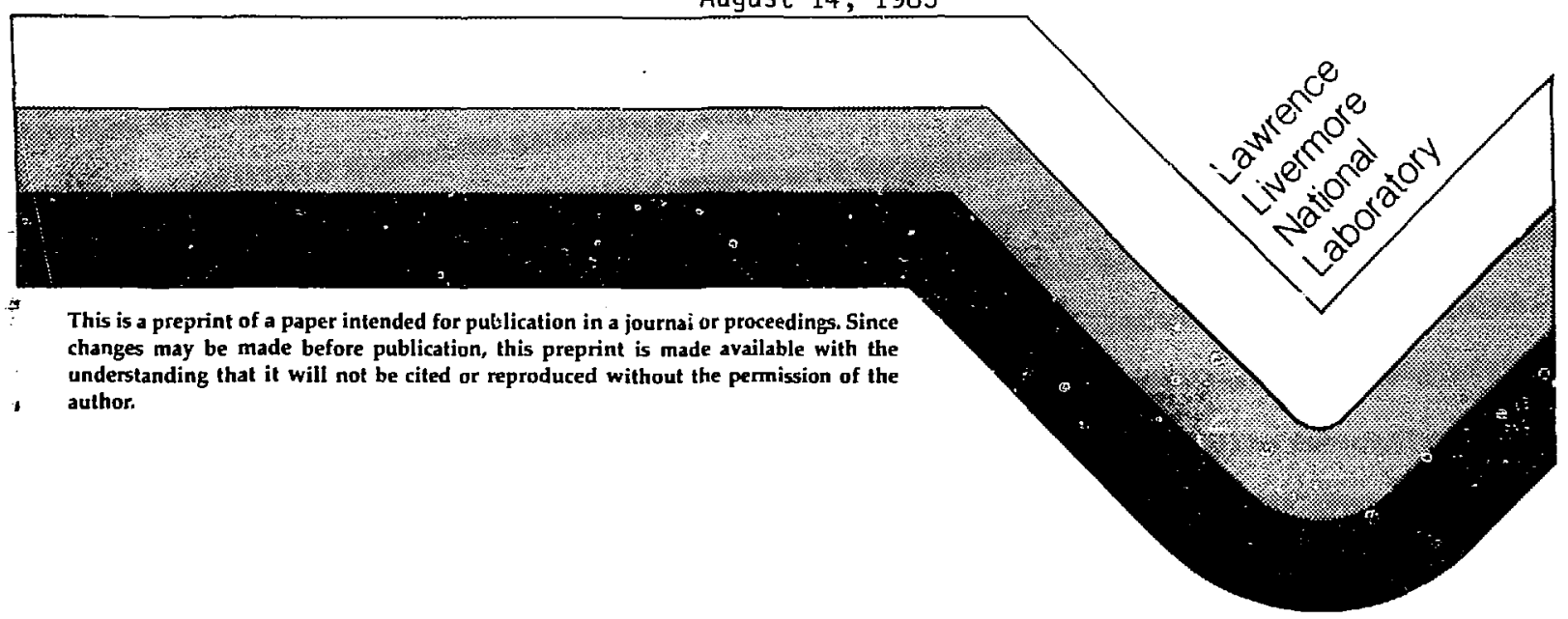




\section{NUAERICAL SIMULATION OF SECONDARY ELECTRON ORBITS NEAR AN ELECTRON BEAM PRUPAGATIHG IN A LOW PRESSURE GAS*}

A. R. Teague and S. S. Yu Lawrence Livermore National Laboratory

P.0. Box 808

Livermore, California 94550

August 14, 1985

UCRL- -91956

DE86 001651

ABSTRACT

A numerical simulation has been constructed to obtain a detailed, quantitative estimate of the electromagnetic fields generated in a recently-proposed collective accelerator scheme for electrons. The code treats the secondary electrons by particle simulation and the beam dynamics by a time-dependent envelope model. The simulation gives a fully relativistic description of secondary electrons moving in selfconsistent electromagnetic fie?ds. The calculations are made using coordinates $t, x, y, z$ for the electrons and $t$, ct-z, $r$ for the axisymetric electromagnetic fields and currents. Code results showing the axial electric field dependence on the configuration of the ultrashort U.V. laser pulse will be given.

\footnotetext{
* Work performed jointly under the auspices of the U. S. Department of Energy by

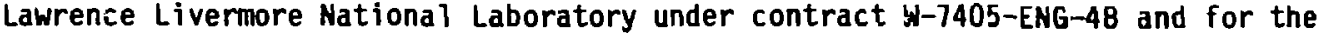
Department of Defense under Defense Advanced Research Projects Agency ARPA Order No. 4395 Amendment No. 31, monitored by Naval Surface Weapons Center under under document number N60921-85-P0W0001; and SDIO/BMD-ATC MIPR No. W3-RPD-53-A127.
} 


\section{OVERVIEW OF A COLLECTIVE ACCELERATOR SCHEME}

It has been proposed [1] to Lse a high-current, medium-energy electron beam as a "charging beam" to accelerate and focus a short, high-energy electron bunch. The accelerator would have very high accelerating field gradients (of order several $\mathrm{MeV} / \mathrm{cm}$ ) with experimentally useful luminosity and high efficiency in transferring energy from the charging beam to the high-energy beam. No quadrupole magnets are required for focusing either the charging beam or the high-energy beam.

Potential energy for the high-energy beam conses from a coiumn of unneutralized ions, produced by the charging beam in three steps. (1) Laser guiding [2] is used to established the beam axis through the accelerator. Direct beam ionization of a low-pressure high-molecular weight background gas establishes propagation in the ion focused regime (IFR). In this mode, secondary electrons generated by the charging beam are expelled to the accelerator wall by the beam electrostatic field in a time short compared with the pulse length of the charging beam, and the remaining ions focus and partially neutralize the beam electrons. Ion charge densities comparable in magnitude to charging beam densities are produced near the tail of the charging beam. (3) The charging beam pulse is cut off abruptly at the tail, leaving an ion column wake which produces a large positive radial electric field (of order $M e V / c m$ ). The proposed accelerator scheme uses this intense radial electric field to generate a negative axial accelerating field of comparable intensity, Before the iens can disperse (which takes on the order of $1 \mathrm{~ns})$, a second laser with a pulse length in the picosecond range creates a phototonization plasma whose electrons rush radially inward, creating a 
negative $E_{Z}$ "spike" following the charging beam. The high-energy electron bunch is accelerated in the $E_{Z}$ spike region, which simultaneousiy has a very large focusing pinch field $E_{r}-B_{\theta}$.

\section{SELF-CONSISTENT SIMULATION OF THE ACCELERATOR}

All beam associated fields, currents, and boundary conditions are assumed axisymmetric. The charging beam and high-energy bunch are described by an envelope model [3], which determines beam radius, enargy, and emittance as functions of axial position and time. Secondary electrons are generated through direct ionization in the charging beam body and through photoionization by the guiding laser and by the picosecond laser at the tail of the charging beam. Trajectories of all these electrons are calculated by integration of the relativistic equations of motion for particies in electric and magnetic fields. The ion motion is always non-relativistic and is essentially only radial motion in the potential well of the IFR channel or in the repulsive collective radial electric field after the charging beam passes. Finally, the electromagnetic fields of the beam and secondaries are described by an approximate form of Maxwell's equations in which, with proper. choice of beam related coordinates, time appears as a parameter rather than ail independent variable (see Eqs. (5-7)).

\section{CALCULATION OF ELECTROHAGNETIC FIELDS AND SECONDARY ELECTRON ORBITS}

We consider the time evolution of a system consisting of beam associated electromagnetic fields $E$ and B satisfying Maxwell's equatiors 


$$
\begin{aligned}
& \frac{1}{c} \frac{\partial \vec{E}}{\partial t}=\nabla \times \vec{B}-\frac{4 \pi}{c} \vec{J} \\
& \frac{1}{c} \frac{\partial \vec{B}}{\partial t}=-\nabla \times \vec{E}
\end{aligned}
$$

and $N$ secondary electrons with pesitions $\vec{r}_{j}$ and velocities $\vec{v}_{j}$ satisfying the relativistic equations of motion $(j=1, N)$

$$
\frac{d}{d t} \frac{\vec{v}_{j}}{\left(1-v_{j}{ }^{2} / c^{2}\right)^{1 / 2}}=\frac{e}{m}\left[\vec{E}\left(r_{j}, t\right)+\frac{\vec{v}_{j}}{c} \times \vec{B}\left(\vec{r}_{j}, t\right)\right] .
$$

(The two divergence equations $\nabla \cdot \vec{E}=4 \pi p$ and $\nabla \cdot \vec{B}=0$ are imposed as initial conditions.) The current $J$ in Eq. (2) has contributions from secondary and beam electrons and ions.

Suppose the beam propagates in the $+z$ direction. If the currents and boundary conditions are axisymmetric, then all field quantities depend only on $r=\left(x^{2}+y^{2}\right)^{1 / 2}, z$, and $t$. However, it is convenient to use as independent variables $r, \zeta$, and $t$, where

$$
\zeta=c t-z
$$

is the (positive) distance behind the beam front. In the axisymmetric case a closed set of equations may be obtained for the three field components $E_{r}$, $E_{z}, B_{\theta}$ and the two current components $J_{r}$ and $J_{z}$. For beam particles with axial velocities essentially equal to $c, \partial / a t \ll c a / \partial \zeta$ for any field quantity. In the limit that a/at terms are negligibly small. Maxwell's equations become 


$$
\begin{aligned}
& \frac{\partial}{\partial r} E_{z}=\frac{4 \pi}{c} J_{r} \\
& \frac{\partial}{\partial \zeta}\left(E_{r}-B_{\theta}\right)=-\frac{4 \pi}{c} J_{r} \\
& \frac{1}{r} \frac{\partial}{\partial r} r B_{\theta}=\frac{4 \pi}{c} J_{z}+\frac{\partial}{\partial \zeta} E_{z} .
\end{aligned}
$$

All fields and currents in Eqs. (5-7) are functions of $r, \zeta, t$. However, $t$ is no longer an independent variable in the field equations but rather a parameter: the fields at time $t$ are produced solely by the currents at time t. Time is, of course, the appropriate independent variable for secondary particle motion. As the particles move the associated currents are generally time-dependent, which injects a parametric time-dependence into the fielus via Eqs. (5-7). (Any external accelerating and focusing fields affect the secondary electron motion through Eq. (3), but the external fields are not included in Eqs. (5-7).)

Since the ions are nonrelativistic and respond essentially only to $E_{r}$ it is inappropriate to treat the ion motion with the relativistic equation of motion Eq. (3) and the complete Haxwell's equations Eqs. (1-2). Accordingly ions are treated by the non-relativistir. equation of motion

$$
\frac{d}{d t} \vec{r}_{j}=\frac{q_{j o n}}{m_{i o n}} \vec{E}_{r}\left(\vec{r}_{j}\right)
$$

where $E_{r}$ is calculated from the ion and electron charge densities $\rho_{j}$ and $P_{e}$ using Poisson's equation

$$
\frac{1}{r} \frac{\partial}{\partial r} r E_{r}=4 \pi\left[\rho_{i}+\rho_{e}\right] \text {. }
$$




\section{SIMULATION DETAILS AND RESULTS}

The field equations (5-7) are solved implicitly in $r$ and $\zeta$. To obtain a fully centered differencing scheme for Eqs. (5-7), four separate interlaced grids are used, and non-linear gridding in $r$ is used to increase the number of points near the beam axis. Appropriate boundary conditions on the fields are specified on the beam axis, beam wall, and beam head. The two-dimenstonal array is protected on all four sides by guard cells so that physical particles always lie in interior cells of the fleld array and never on edge cells. A completely relativistic particle push [4] valid for arbitrary electric and magnetic fields is used.

For the results shown in this paper, the $\zeta-r$ grid was $101 \times 31$, and secondary electrons were represented by 5000 macro-particles having the electron charge/mass ratio. The code requires around 10 seconds (dependent on graphics requested) of CRAY I computer time to advance the complete system 200 time steps.

Figures 1-5 show results of a typical simulation. The ion column had a radius of $1 \mathrm{~mm}$, while the picosecond laser photoionization channel had a radius of $3 \mathrm{~mm}$. The $E_{z}$ spike is evident in $\mathrm{Fig} .1$ and lies about $1.5 \mathrm{~mm}$ behind the tail of the picosecond laser. All quantities in the figure are in cgs units. The peak $E_{2}$ spike of around -4000 statvolts $/ \mathrm{cm}$ corresponds to $1.2 \times 10^{8}$ volts/meter. The high-energy bunch has length $0.5 \mathrm{~mm}$ and radius $10 \mu \mathrm{m}$. In this case, oscillations occur after the $E_{z}$ spike because the high-energy pulse loes not load the accelerating $E_{2}$ field optimally. The photoionization electrons do not lose most of their energy to the high-energy 
bunch and therefore oscillate radially about beam axis. For al? figures the particles and fields were advanced two hundred time steps for a total of 33 picoseconds.

Work is in progress to optimize the accelerator luminosity and efficiency by varying the beam and photoionization channel profiles by improving the loading of the accelerating field.

\section{FIGURES}

Fig. 1. Axial accelerating field $E_{z}$ in statvolts/cm. (All quantities in Figs. (1-5) are in cgs units.)

Fig. 2. Radial electric field at $r=1 \mathrm{~mm}$.

Fig. 3. Pinch field $E_{r}-B_{\theta}$ at $r=1 \mathrm{~mm}$.

Fig. 4. $B_{\theta}$ at $r=1 \mathrm{~mm}$.

Fig. 5. Positions of 5000 (macro) electrons after 33 picoseconds. 

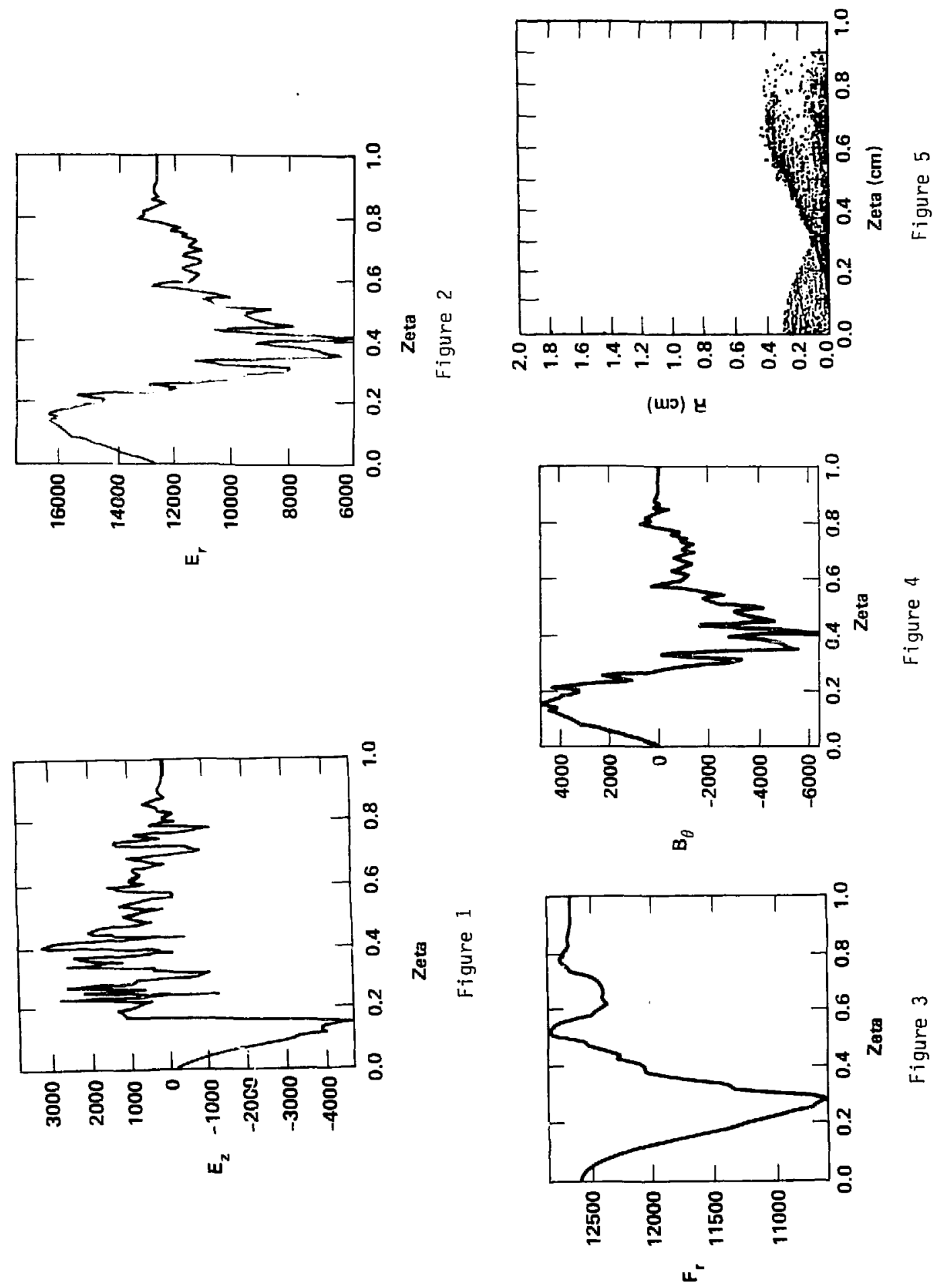


\section{REFERENCES}

[1] R. J. Brigys, "A collective Accelerator for Electrons," 1985 Particle Accelerator Corference, paper VA5, May 1985.

[2] W. E. Martin, G. J. Caporaso, W. M. Fawley, D. Prosnitz, and A. G. Cole," Electron Beam Guiding and Phase-Mix Damping by a laser-Ionized Channel," Phys. Rev. Lett. 54, 685-688 (1985).

[3] E. P. Lee and R. K. Cooper, "General Envelope Equations for Cylindrically Symetric Charged-Particle Beams," Particle Acrelerators 1 , 83-95 (1976).

[4] C. K. Birdsall and A. B. Langdon, Plasma Physics Via Computer simulation, Mc Graw-Hil1, New York 1985. Section 15-4 describes a version (which is used in our simulation) of the relativistic particle push developed by $\mathrm{J}$. Boris (1970).

\section{ACKNOWLEDGMENT}

The Authors thank J. K. Boyd, R. J. Briggs, L. R. Moreno, and E. T. Scharlemann for their contribution to this paper.

MRT : emj

9627v

\section{DISCLAIMER}

This report was prepared as an account of work sponsored by an agency of the United States Government. Neither the Urited States Government nor any agency thereof, nor any of their employees, makes any warranty, express or implied, or assumes any legal liability or responsibility fo: the accuracy, completeness, or usefulness of any information, apparatus, product, or process diselosed, or represents that its use would not infringe privately owned rights. Reference herein to sny specific commercial product, process, or service by trade name, trademark, manufacturer, at otherwise does not necessarily constitute or imply its endorsement, recommendation, or favoring by the United States Goverament or any agency thereof. The views and opinions of authors expressed herein do not necessarily state or reflect these of the United States Government or any agency thereof. 